\title{
NOTE ON TIMESTAMPS
}

In order to help the reader locate media sequences discussed, I have included timestamps throughout the text, notes, and figures. I preface these timestamps with the abbreviation "ca." (circa) because the digital time counts given for the location of specific frames may be imprecise. Different copies exist of the same moving image works, with some different opening sequences, and also occasionally edited differently. Such variation can alter the time countsometimes just a second or two, but occasionally up to a minute and a half. 